\title{
Research Paper \\ Effect of Teaching the Components of Spiritual Intelligence on Death Anxiety in the Elderly
}

\section{Aref Majidi $^{1},{ }^{*}$ Omid Moradi $^{1}$}

1. Department of Psychology, Faculty of Education and Psychology, Sanandaj Branch, Islamic Azad University, Sanandaj, Iran.

$\begin{gathered}\text { use your device to scan } \\ \text { and read the article online }\end{gathered}$
Citation: Majidi A, Moradi O. [Effect of Teaching the Components of Spiritual Intelligence on Death Anxiety in the Elderly
(Persian)]. Iranian Journal of Ageing. 2018; 13(1):110-123. https://doi.org/10.21859/SIJA.13.1.110

Received: 07 Sep. 2017 Accepted: 08 Jan. 2018

Key words: Spiritual intelligence, Death anxiety, Old people

\section{A B S TR ACT}

Objectives This study aimed at studying the effect of teaching spiritual intelligence principles on death anxiety among senior citizens living in retirement homes of Sanandaj City.

Methods \& Materials This study was a practical and quasi-experimental research performed by pre- and posttesting the control and experimental groups. The statistical population comprised all elderly residents of retirement homes in Sanandaj City. Twenty subjects were selected by convenient sampling from among the individuals who were willing to participate in the study and who received a high score in the death anxiety scale. They were then equally divided into two groups (10 subjects per group). The fear of death scale devised by Collet- Lester (1969) was used for pre-testing the subjects. The members of the experimental group received Spiritual Intelligence-based intervention in eight 90-minute sessions. The control group received no such intervention during this period. After the intervention, all the subjects were post-tested. The collected data were analyzed by the one-way and multi-way analysis of variance (ANOVA) and the SPSS-22 software.

Results The mean and standard deviation of the age of the experimental group was $62.5 \pm 5.68$ years and control group was $63.1 \pm 4.33$ years. The results of covariance analysis showed a significant difference in post-test between the experimental group $(120.9 \pm 4.81)$ and the control group $(131.3 \pm 8.65)(P \leq 0.01)$. So, the results indicate that educating the subjects about spiritual intelligence components reduced their death anxiety $(P \leq 0.01)$. The intervention also reduced the death of others and dying of others dimensions in the subjects $(P \leq 0.01)$, but no significant impact was observed for the death of self and dying of self dimensions.

Conclusion According to the findings, teaching spiritual intelligence principles reduces death anxiety. Therefore, it is recommended that more attention should be paid to teaching spiritual aspects in retirement centers.

\section{Extended Abstract}

\section{Objectives}

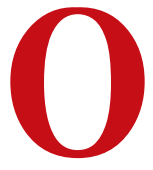

ne of the most common mental disorders of old age is death anxiety, which means predicting your death and fear of the death of important people in life [1]. Such feelings can lead to helplessness, physical changes, loss of control, feeling lonely and guilty and can affect the functions of ordinary life of the individuals [2]. Therefore, it is necessary to identify the factors responsible for reducing death anxiety. Given this background, this study aimed to investigate the effect of teaching the components of spiritual intelligence on the amount of death anxiety in the elderly living in Sanandaj's nursing homes. To achieve this goal, two questions were considered and examined: 1)

\section{* Corresponding Author:}

Omid Moradi, PhD

Address: Department of Psychology, Faculty of Education and Psychology, Sanandaj Branch, Islamic Azad University, Sanandaj, Iran.

Tel: +98 (918) 8721606

E-mail: moradioma@gmail.com 
Is the training of spiritual intelligence components effective in reducing death anxiety?; and 2) Does learning the components of spiritual intelligence reduce the dimensions of death anxiety (death of yourself, seeing you are dying, death of your neighbors, seeing dying relatives)?

\section{Methods and Materials}

This study is applied research in terms of purpose, and it is semi-pilot with pre-test-post-test and control group in terms of implementation method. The statistical population of the research was all people living in nursing homes in Sanandaj in the first half of 2016. The sample selection process was as follows. After obtaining the necessary legal permissions, 100 Death Anxiety Inventory questionnaires were distributed among the elderly people with the aim of screening. Those who had an average score for their death anxiety in two higher deciles were recognized as eligible subjects. Eventually, these 20 subjects were placed randomly in two experimental and control groups. The inclusion criteria were having reading and writing skills, lack of organ defects in the auditory, speaking and visionary organs, not having acute cognitive impairment, the desire to participate in the training component of spiritual intelligence, and a high score on death anxiety. It should be noted that during the sample selection, ethical considerations such as freedom of participation or non-participation in research, anonymous question- naire, obtaining legal licenses, and obtaining moral confirmation were observed. This research was approved by the Ethics Committee of Islamic Azad University, Sanandaj Branch.

After selecting the sample subjects, the Collett-Lester's Fear of Death Scale (1969) was distributed as a pre-test on subjects. This scale included 32 questions and 4 subscales of death of the self, seeing own death, the death of surroundings, and seeing neighbors dying. Then the experimental intervention group received the training approach on spiritual intelligence concepts in 8 sessions for 90 minutes. During this time, the control group did not receive any intervention. After the end of the intervention sessions, the posttest was performed on subjects. Using one-way covariance analysis and multimode analysis and SPSS22 software, the collected data were analyzed.

\section{Results}

The mean and standard deviation of the age of the experimental group was $62.5 \pm 5.62$ years and for the control group was $63.1 \pm 4.3$ years. In Table 1 , the mean and standard deviation of the subjects' death anxiety scores are presented in the pre-test and post-test phase.

After providing the descriptive findings, inferential analysis of data is presented. The normal distribution of data was examined through Shapiro-Wilk's test. Ac-

Table 1. Mean and standard deviations of anxiety scores for subjects

\begin{tabular}{|c|c|c|c|c|c|}
\hline \multirow{2}{*}{ Variable } & \multirow{2}{*}{ Group } & \multicolumn{2}{|c|}{ Pretest $(\mathrm{N}=10)$} & \multicolumn{2}{|c|}{ Post-test (N=10) } \\
\hline & & Mean & SD & Mean & SD \\
\hline \multirow{2}{*}{ Death of the se } & Experimental & 34.8 & 3.08 & 32.3 & 2.11 \\
\hline & Control & 33.7 & 4.06 & 33.3 & 2.67 \\
\hline \multirow{2}{*}{ Seeing the self $\mathrm{c}$} & Experimental & 32.5 & 2.72 & 30.4 & 1.96 \\
\hline & Control & 32.4 & 1.9 & 32.8 & 2.35 \\
\hline \multirow{2}{*}{ Death of compa } & Experimental & 35.2 & 2.62 & 28.4 & 2.32 \\
\hline & Control & 35.3 & 3.23 & 32.1 & 3.84 \\
\hline \multirow{2}{*}{ Seeing dying comp } & Experimental & 34.6 & 1.71 & 29.8 & 1.75 \\
\hline & Control & 33.9 & 1.66 & 33.1 & 3.54 \\
\hline \multirow{2}{*}{ Total score of death anxiety } & Experimental & 137.1 & 8.13 & 120.9 & 4.81 \\
\hline & Control & 135.3 & 7.82 & 131.3 & 8.65 \\
\hline
\end{tabular}


cordingly, the amount of Shapiro-Wilk's statistic on the death anxiety score in the pre-test equaled to 0.927 , and the level of significance was 0.136. The amount of Shapiro-Wilk's statistic on Death Anxiety Rating in post-test equaled to 0.922 , and the level of significance was 0.109 . Due to the lack of significant results and confirmation of zero assumption, it was ensured that data distribution is normal. Therefore, parametric tests were used for data analysis (Table 1).

To check the first research question, one-way covariance analysis was used. The results of the first question showed that the effect of independent variables on the training of spiritual intelligence components on the reduction of the total death score of the elderly was statistically significant $(\mathrm{F}=27.053$ and $\mathrm{Sig} .=0.001)$. The post-test mean scores of the experimental group were found to be decreased compared to the control group. Therefore, it can be said that learning the components of spiritual intelligence could significantly reduce the total death anxiety in the experimental group compared to the control group.

Multivariate covariance analysis was used to investigate the second question. Based on the results, the values of $\mathrm{F}$ calculated in the case of 4 tests of Piley effect, Wilks Lambda, Hoteling effect, and the Roy's Largest Root Test were significant at the level of 0.01 . Therefore, it was found that there is at least a significant difference between the two pairs. The main results of the covariance analysis also showed that the values of $\mathrm{F}$ calculated in group variables in the dimensions of death of relatives and seeing dying companions $(F=6.727,27.675)$ are significant at the level of $\mathrm{P} \leq 0.05$. But these values were not significant in terms of death of the self and seeing themselves dying. Significance in subscales of death of relatives and seeing dying companions means that the training of spiritual intelligence components has been successful. These two subscales in the test group are reduced compared to the control group.

\section{Conclusion}

The results of this study showed that learning the components of spiritual intelligence reduces the extent of death anxiety (deaths of companions and seeing relatives dying) in the elderly. However, there were no significant results in reducing the dimensions of death anxiety such as death of the self and seeing themselves dying. These results were consistent with previous research findings [3-7].
The limitation of this research was the lack of longterm follow-up of the training results because of the time limit for the research. Therefore, it is recommended that future studies should focus on long-term implications of interventions based on spiritual intelligence training. Overall, considering the findings of the research, it is recommended that training courses on spiritual intelligence components should be held in nursing homes, hospitals, and similar environments where patients and helpless people reside.

\section{Acknowledgments}

This research was extracted from the second author's MA thesis in the Department of Psychology, Faculty of Education and Psychology, Sanandaj Branch, Islamic Azad University, Sanandaj, Iran.

\section{Conflict of Interest}

The authors declared no conflicts of interest. 


\title{
بررسى تأثير آموزش مؤلفهاى هوش معنوى بر ميزان اضطراب مرگى در سالمندان
}

\author{
عارف مجيدى'، "اميد مرادى'
}

1- كروه روانشناسى، دانشكده علوم تربيتى و روانشئاسى، واحد سنيدج، دانشكاه آزادا سلامى، سنندج، ايران.

\section{حكSי}

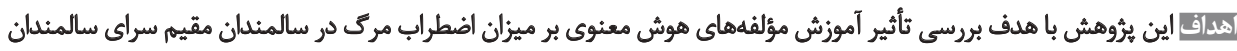

$$
\text { شُهر سيثلنج ائجام شُد. }
$$

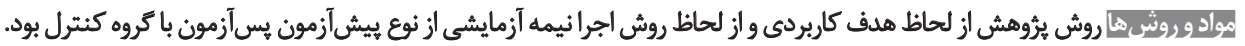

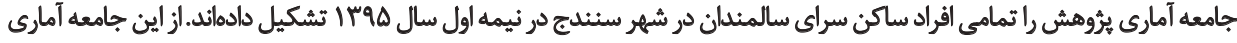

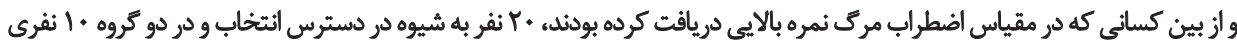

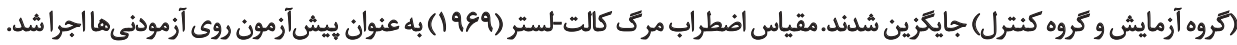

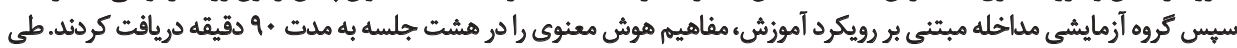

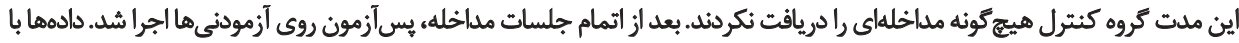

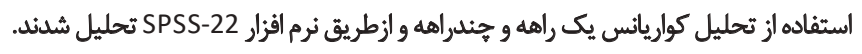
ياقته ها ميانتين و انحراف معيار سنى كروه آزمايش

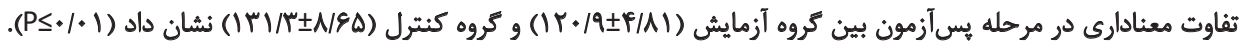

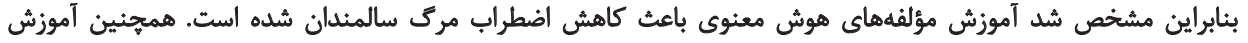

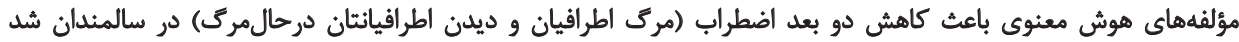

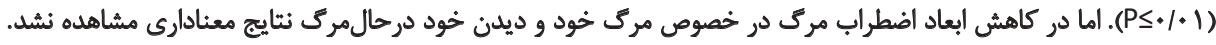

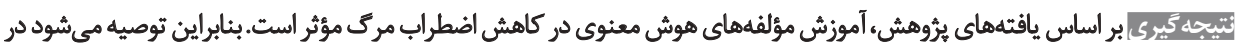

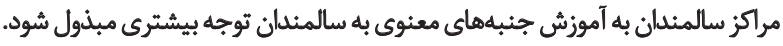

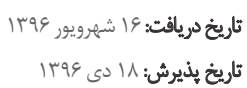

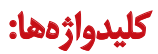

هوش معنوى، اضطراب

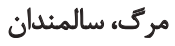

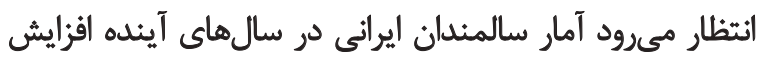

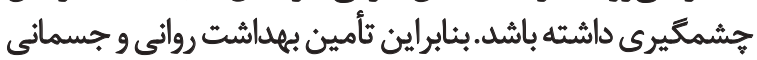

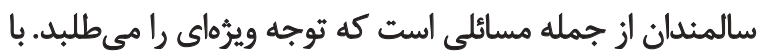

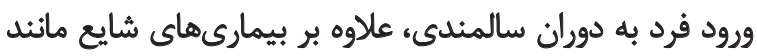

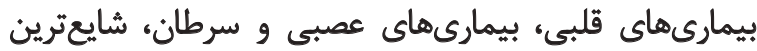

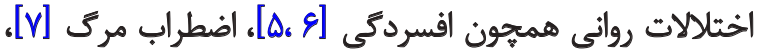

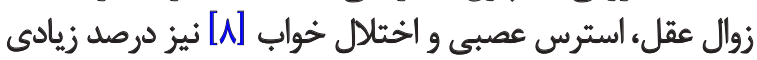

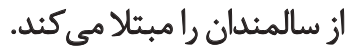

يكى از شايعترين اختلالات روانى دوران سالمندى، اضطراب

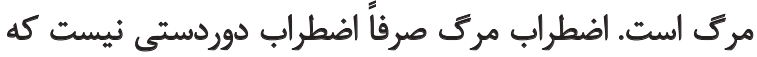

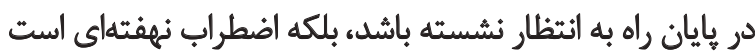

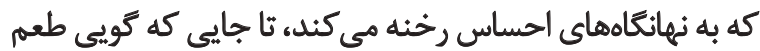

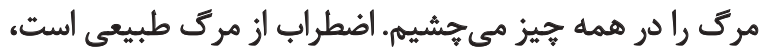

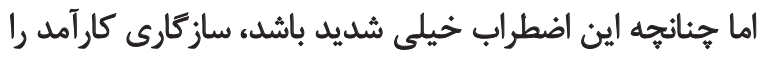

با بيشرفت و كسترش علوم بهداشتى، هر سال بر شمار

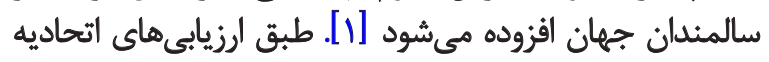

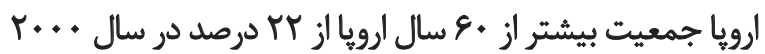

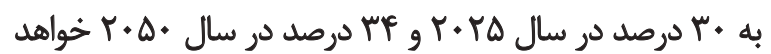

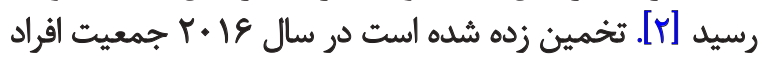

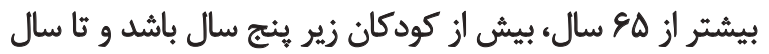

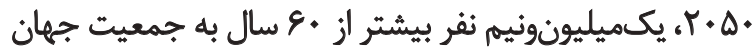

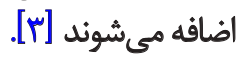
با توجه به افزايش شاخص اميد به زندكى در جهان و ايران،

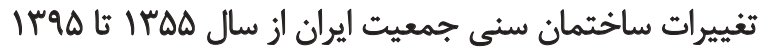

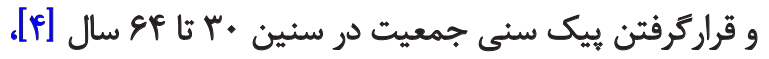




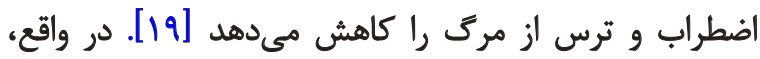

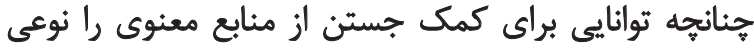

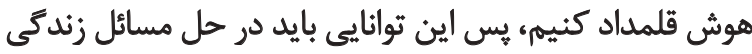

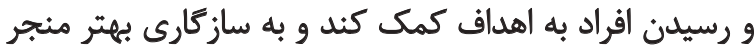

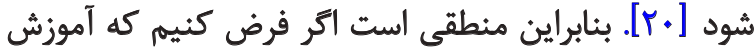

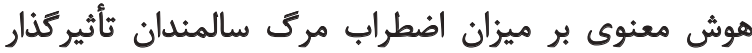

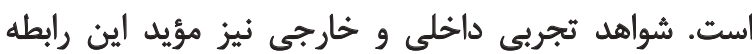

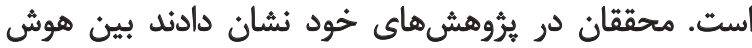

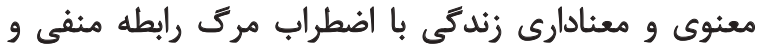

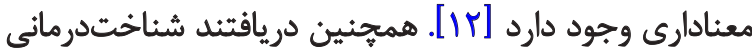

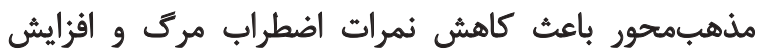

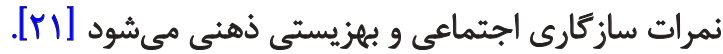
علاوه بر شواهد تجربي داخلى، نتايج تحقيقات خارجى نيز

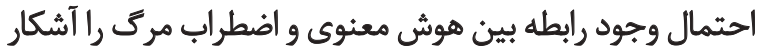

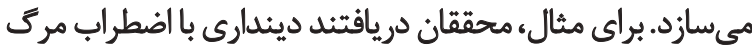

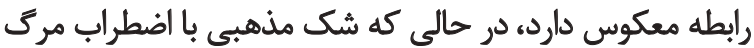

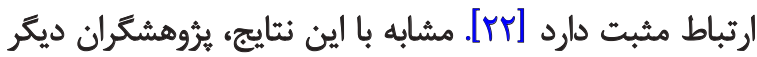

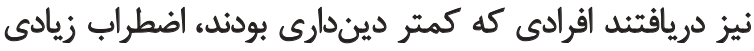

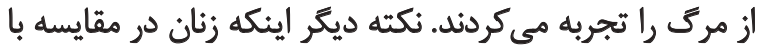

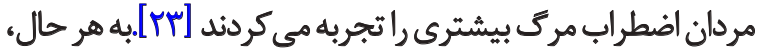

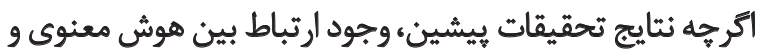

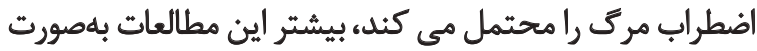

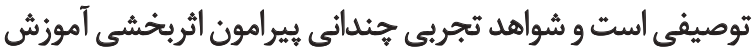

هوش معنوى بر كاهش اضطراب مرك در دسترس نيست.

با توجه به خلأ بروهشى موجود، هدف اصلى تحقيق حاضر،

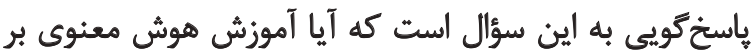

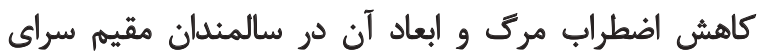

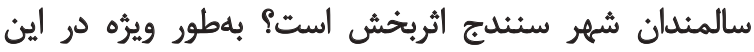

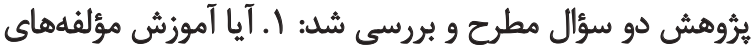

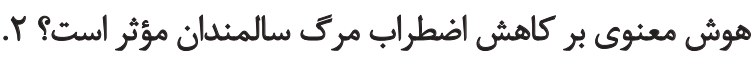

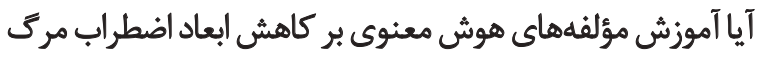

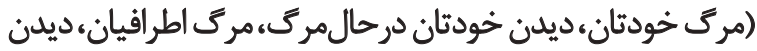
اطرافيانتان در حال مركى) سالمندان مؤثر است؟

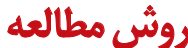

يروهش حاضر از لحاظ هدف كاربردى و از لحاظ روش

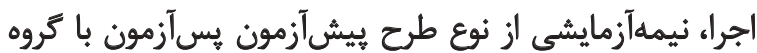

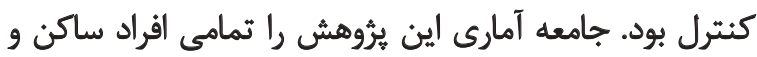

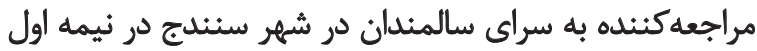

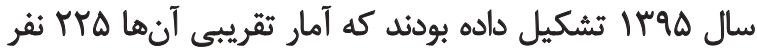

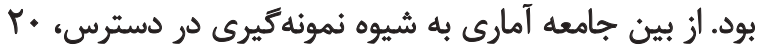

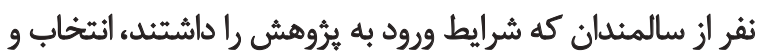

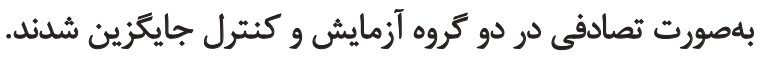

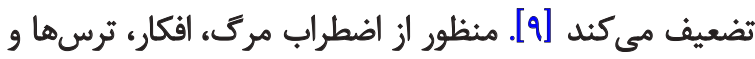

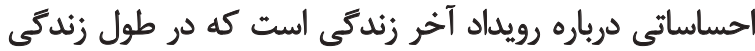

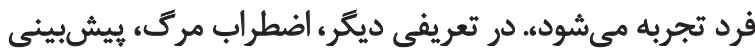

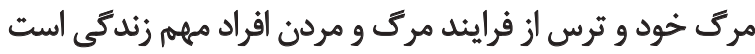

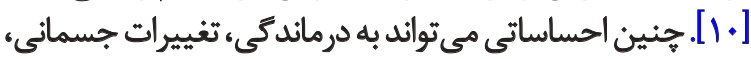

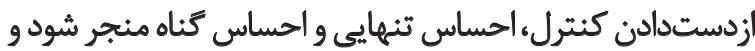

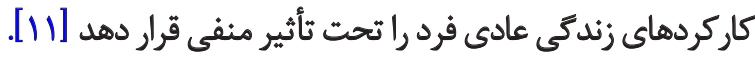

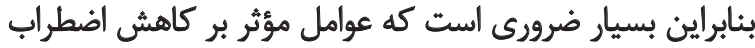

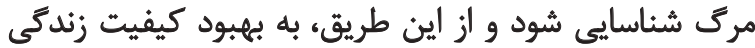

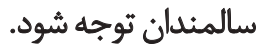

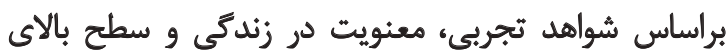

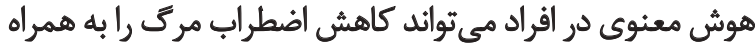

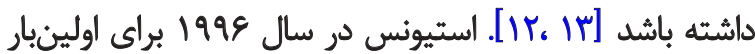

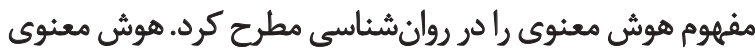

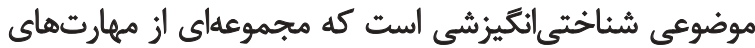

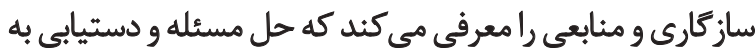

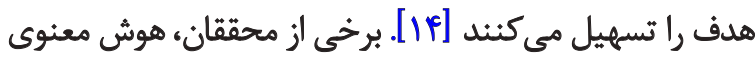

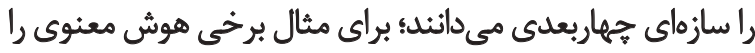

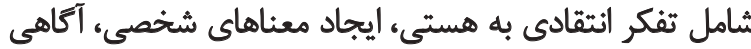

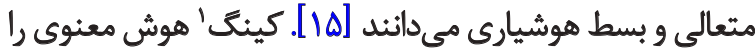

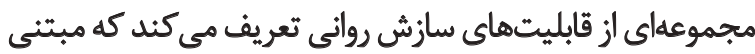

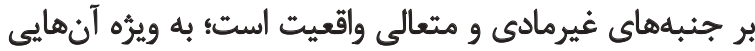

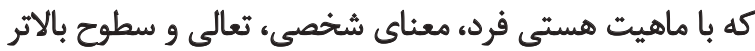

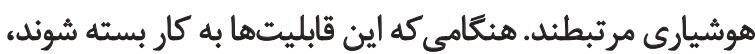

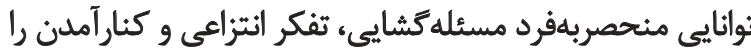

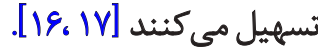

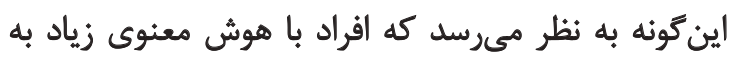

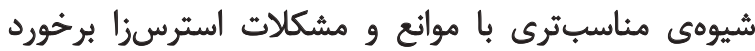

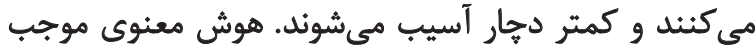

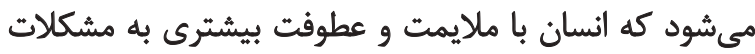

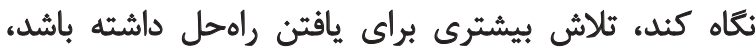

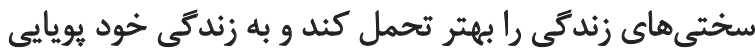

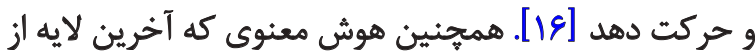

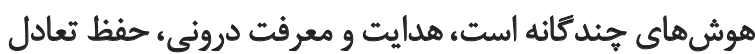

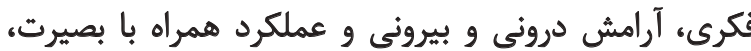
ملايمت و مهربانى را شامل مي شئود

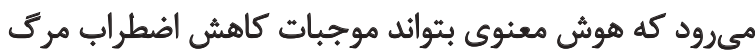

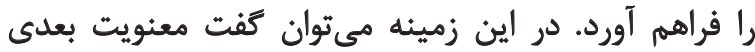

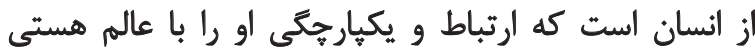

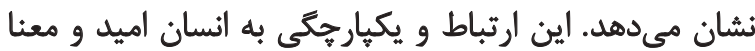

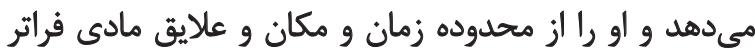

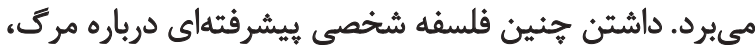

1. King 
جدول ا. جلسات آموزش هوش معنوى

جلسه

آشناييى اعضا با يكديكر، اجراى ييش آزمون و شح هوش معنوى، ارائه توضيحاتي اوليه درباره مفهوم اضطراب و اضطراب مرى

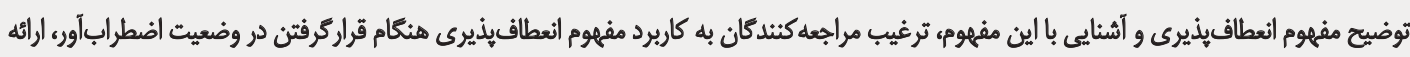

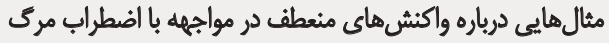

تشويق افراد براي زير سؤالبردن عقايد متعارف خود دوباره جهان و تجربيات، كمى به افراد براى هذيرش باورهاى منطقى تر درباره مرى و جهان يس از مرى به منظور كاهش اضطراب مرى

أموزش ارتقاى ثفكر وجودى ائتقادى، طح سؤالاتى درباره ماهيت مرك، ماهيت هستى، فلسفه زندكى، هدف از آفريشش و غيره، كمك به افراد براى ارتقاى اهداف متعالى تر در زندكّى

كمك به فرد در يافتن معناى زندكى، تصويرسازى جديلى از دليل و هدف از زندكى، فراهمركردن بسترى براى تسهيمه باورهاي درست مراجعلكنتدكان

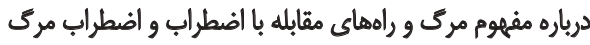

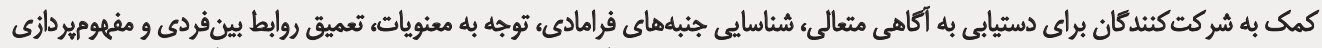

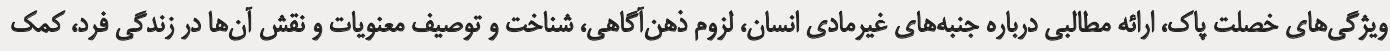

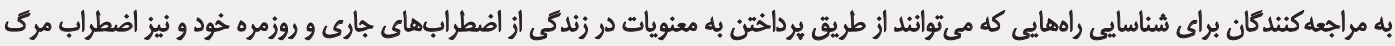
بكاهند

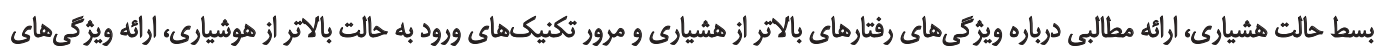

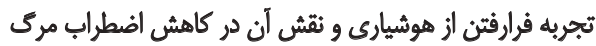

筧

يرؤهى فرم تجديدنظرشده اين مقياس را بر روى نمونهاى به إنهان

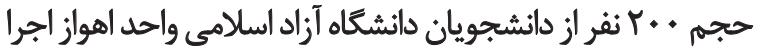

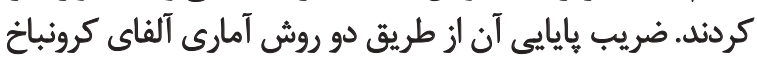

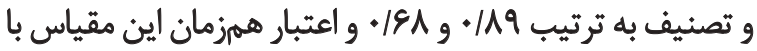

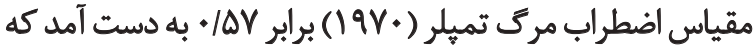

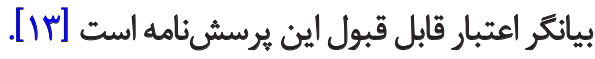
اين مقياس شامل r ب سؤال و جهار خردهمقياس مركى

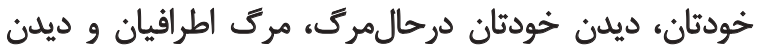

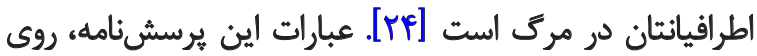

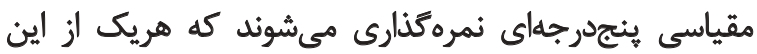

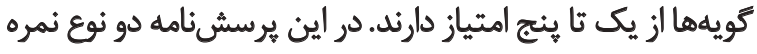

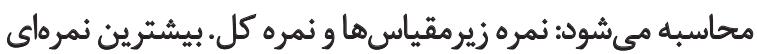

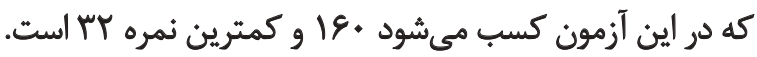

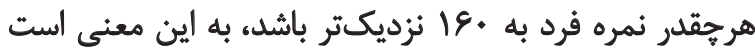

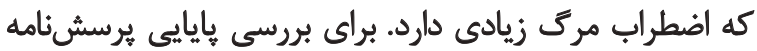

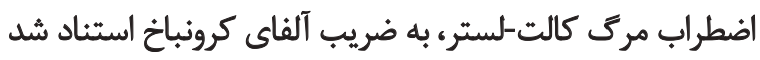

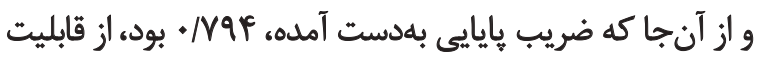
اعتماد ابزار اطمينان حاصل شد. بائ بلد

روند اجراى يثروهش به اين صورت بود كه ابتدا مقياس اضطراب

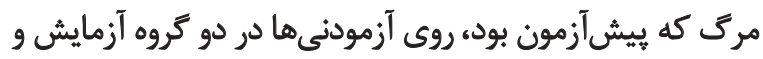

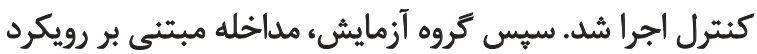

معيارهاى انتخاب افراد نمونه، داشتن سواد خواندن و نوشتن،

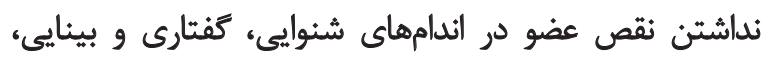

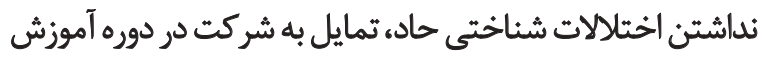

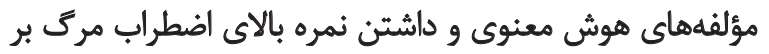
اساس مقياس اضطراب مرى كالت-لستر (1999) بود.

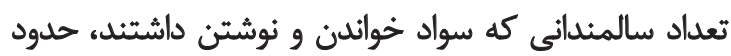

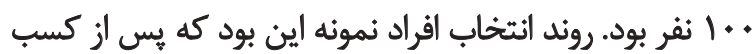

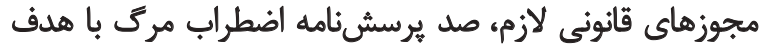

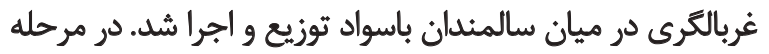

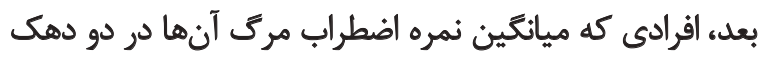

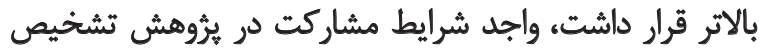

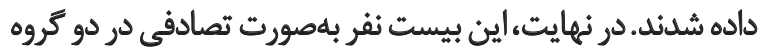

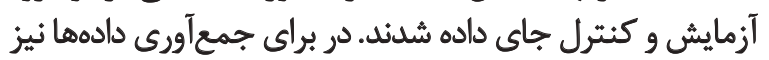

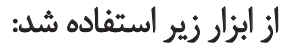

\section{مقياس اضطراب مركّى كالت-لستر 'م)}

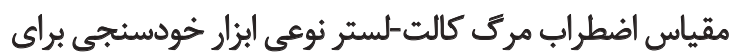

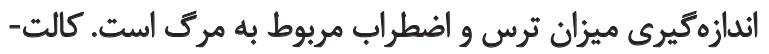

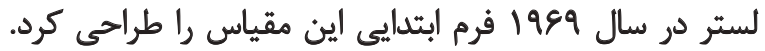

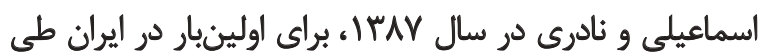

2. Collet - Lester Fear of Death Scale 


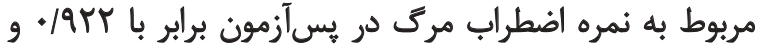

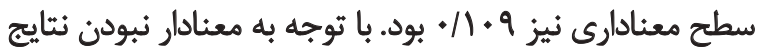

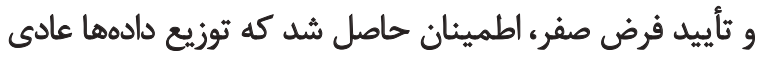

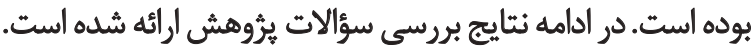
ا. آيا آموزش مؤلفههاى هوش معنوى بر كاهش اضطراب

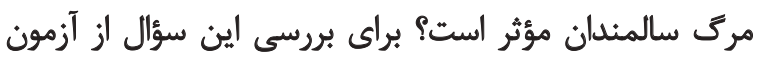

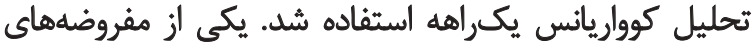

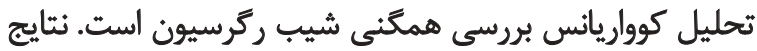

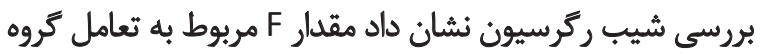

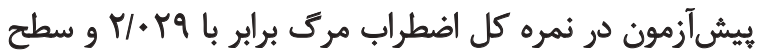

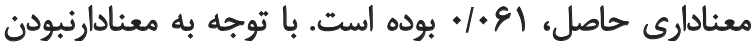

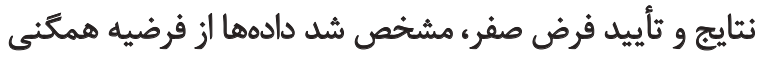

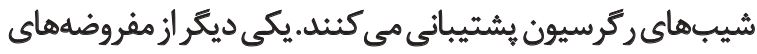

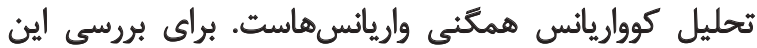

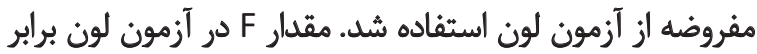

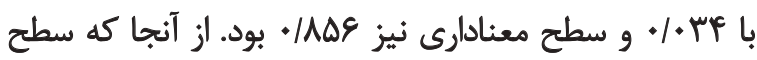

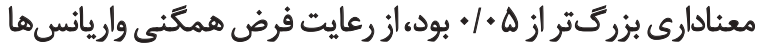

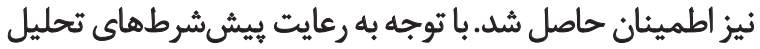
كوواريانس، در جدول شماره f نتايج اين آزمون ارئه بائه شده است.

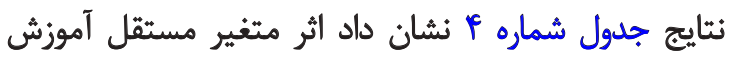

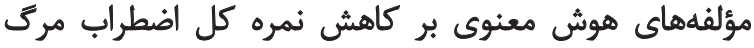

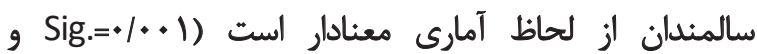

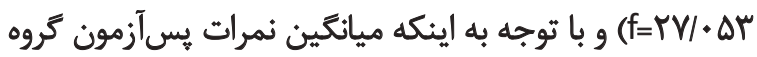

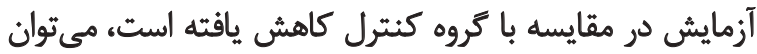

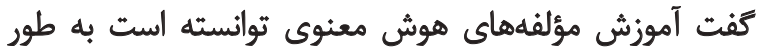

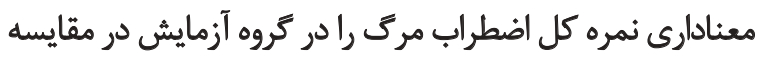

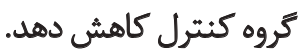

آموزش مفاهيم معنوى را در هشت جلسه •و دوقيقهاي دريافت

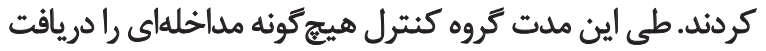

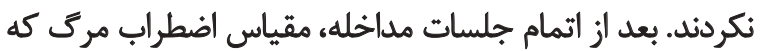

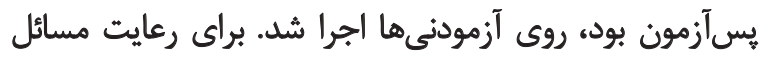

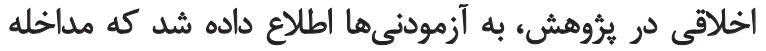

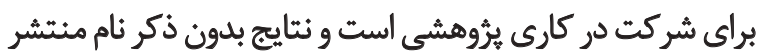

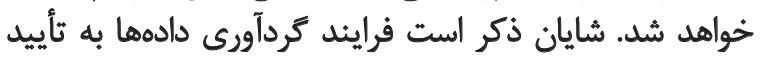

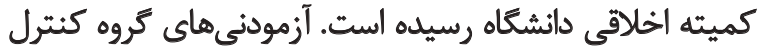
هم تحت مداخلات بدون ساختارى قرار كرفتئد كه از از لحاظ

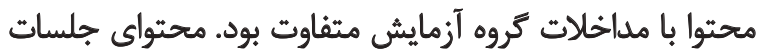

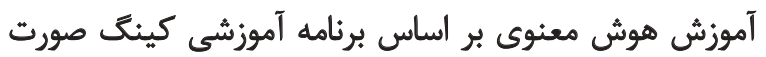

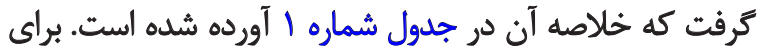

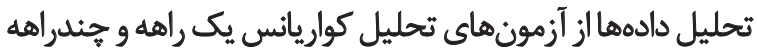

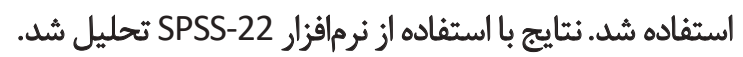

ياقتهها

براساس تحليل اطلاعات جمعيثشناختى ميانكين سنى كروه

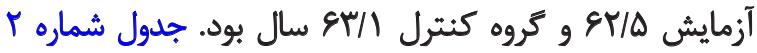

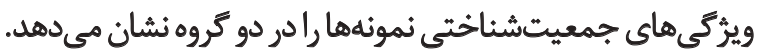

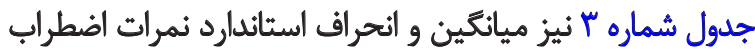

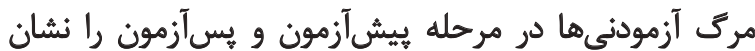

.000

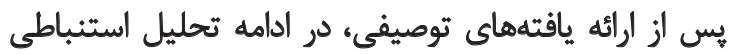

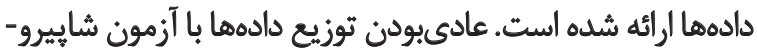

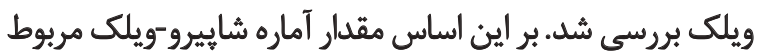

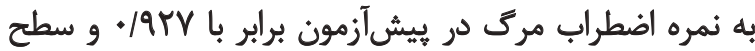

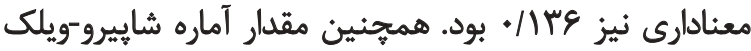

جدول Y. اطلاعات جمعيثشناختي افُراد كروه آزمايش و كنترل

\begin{tabular}{|c|c|c|c|c|c|}
\hline \multicolumn{2}{|c|}{ كروه كتترل (+ (N=) } & \multicolumn{2}{|c|}{ كروه آزمايش (+ (N=1) } & \multirow{2}{*}{\multicolumn{2}{|c|}{ شتغير }} \\
\hline درصد & فراوانى & درصد & فراوانى & & \\
\hline 1. & 1 & 1. & 1 & كارمند & \\
\hline 1. & 1 & 1. & 1 & بيكار & شغل \\
\hline A. & $\wedge$ & $\Lambda$. & $\wedge$ & آزاد & \\
\hline s. & 8 & 8. & 8 & مثاهل & \\
\hline 1. & 1 & $r$. & $r$ & مطلقة & وضعيت تأهل \\
\hline r. & $r$ & r. & r & فوت همسر & \\
\hline 9. & 9 & A. & $\wedge$ & ن ن & $\because \rightarrow$ \\
\hline 1. & 1 & r. & $r$ & مرد & 9 \\
\hline
\end{tabular}

元 
جدول ऍ. ميانكين و انحراف استاندارد نمرات اضطراب مرى آزمودنىها

\begin{tabular}{|c|c|c|c|c|c|}
\hline \multicolumn{2}{|c|}{ يسأزمون (+ (N=1) } & \multicolumn{2}{|c|}{ ييش آزمون (+ (N=) } & \multirow{2}{*}{$\Delta$} & \multirow{2}{*}{ متغير } \\
\hline أنحراق معيار & ميانكين & اتحراق معيار & مياتكين & & \\
\hline$r / 11$ & $M / T$ & $r / * A$ & $m e / s$ & أزمايش & \multirow[b]{2}{*}{ مرى خود } \\
\hline T/EV & 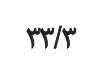 & $1 / .8$ & $\pi / N$ & كتّرل & \\
\hline $1 / 98$ & $r \cdot / f$ & $r / M$ & $M / \Delta$ & آزمايش & \multirow{2}{*}{ ديلن خودثان در حال مرى } \\
\hline T/M & $r / A$ & $1 / 9$ & $M T / P$ & كتترل & \\
\hline T/Mr & rNT & r/EY & $r \Delta / r$ & آزمايش & \multirow{2}{*}{ مركى اطرافيان } \\
\hline r/AP & $M / N$ & $r / r r$ & $r \Delta / r^{\omega}$ & كنترل & \\
\hline I/NA & ra/s & $M$ & $\pi r / s$ & آزمايش & \multirow{2}{*}{ ديدن اطرافيان در حال هرى } \\
\hline$r / \Delta F$ & $m / 1$ & $1 / 89$ & $m / q$ & كتترل & \\
\hline$f / A \mid$ & $1 r \cdot / 9$ & NIr & $|w|$ & أزمايش & \multirow{2}{*}{ نمره كل اضطراب مرى } \\
\hline NFA & $|r| r / r$ & V/Ar & $\mid r \Delta / r$ & كتترل & \\
\hline
\end{tabular}

L

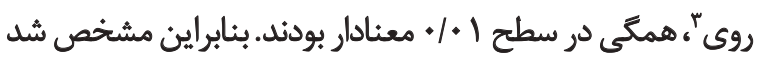
حداقل بين يك جفت ميانغين ثفاوت معنادار وجود دارد.

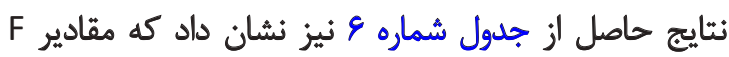

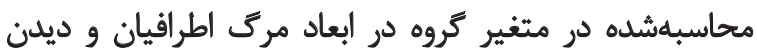

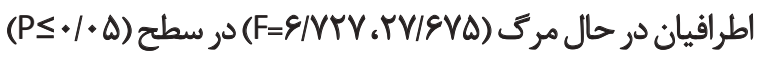

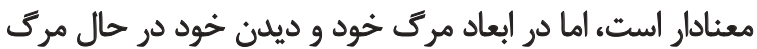

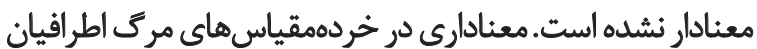

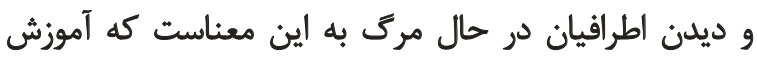

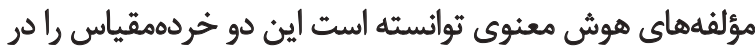

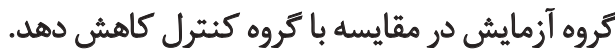

ث

هدف از يروهش حاضر بررسى آموزش مؤلفههاى هوش معنوى

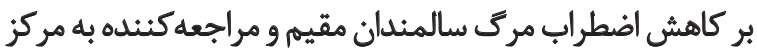

3. Roy Root
T) آيا آموزش مؤلفهاي هوش معنوى بر كاهش ابعاد اضطراب

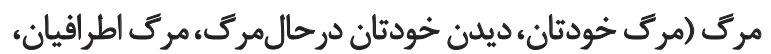

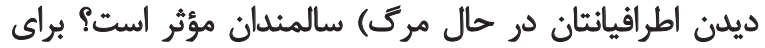

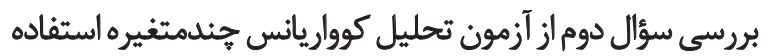

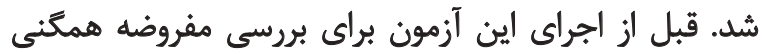

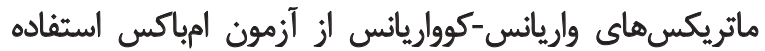

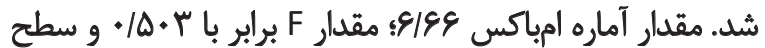

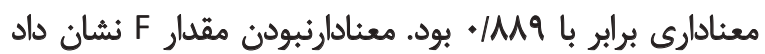
فرض همگنى ماتريكس هاى واريانس-كوواريانس رعايت شده

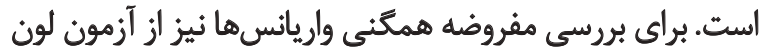

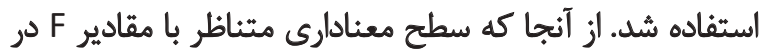

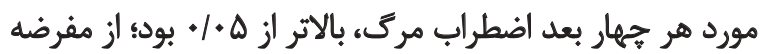

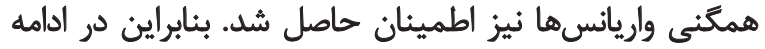

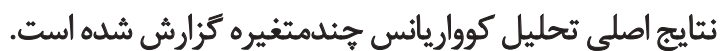
بر اساس نتايج جدول شماره ه، مقادير F محاسبهشده براى جهار

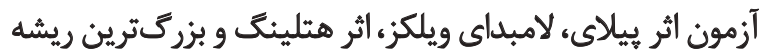

جدول F. تحليل كوواريانس يكراهه براي بررسى معنادارى تثاوتهاي گروهاي آزمايش و كنترل در اضطراب مرى

\begin{tabular}{|c|c|c|c|c|c|c|c|}
\hline ضريب اتا & سطح معنادارى & $F$ & ميانكين مجذورات & $d f$ & مجموع مجذورات & منيع تغييرات & متغير \\
\hline . & $.1 * .1$ & $19 / \mu r+r$ & $F \& 9 / \Delta+F$ & 1 & $F \varepsilon q / \Delta+f$ & ييش أزمون & \\
\hline \multirow[t]{2}{*}{.$|8| f$} & $.1+.1$ & $r V / \cdot \Delta r$ & gQN.. & 1 & gAN..G & كروه & اضطراب مركى \\
\hline & & & repmrm & iv & pirpeq & خططا & \\
\hline
\end{tabular}

ك 
جدول ه. خلاصه نتايج تحليل كواريانس جند متغيره (مانكوا)

\begin{tabular}{|c|c|c|c|c|c|}
\hline سطع معنادارى & df خط & فرضيه df & $F$ & الرزش & نام أزمون \\
\hline$+1+1$ & $r+1 \ldots$ & $N+\cdots$ & G/MTE & I/TAS & اثر ييلايى \\
\hline .10 .1 & rN... & $N \cdots$ & g/us & .1111 & لامبداي ويلكز \\
\hline $.1 . .1$ & $r \& 1 \ldots$ & $N \cdots$ & $V / 8 \cdot 1$ & PIFAY & اثر هتلينك \\
\hline $.1 . .1$ & $10 / \ldots$ & $\%$ & IF/Tr & $r / A 19$ & بزركترين ريشهروى \\
\hline
\end{tabular}

جدول \&. نتايج آزمون تحليل كوواريانس جيندمتغيره بر روى ميانتين نمره هاى يس آزمون ابعاد اضطراب مركى

\begin{tabular}{|c|c|c|c|c|c|c|c|}
\hline ضريب اتا & معنادرإي & $F$ & مجنياتكين مين & df & مجموع مجذورات & مقياس & تغنيبر \\
\hline .1181 & . & r//9Q & glov" & 1 & g/ov" & هرى خود & \\
\hline .118 & 1.18 & $r / r \cdot r$ & $18 / 1 \cdot F^{\circ}$ & 1 & $18 / \Lambda \cdot r^{2}$ & ديلن خود درحال مرى & \\
\hline. legt & .10 .1 & TV/EVO & ITOMFY & 1 & ITA/MPT & مرى اطرافيان & Sروه \\
\hline \multirow[t]{9}{*}{. MTS } & $+1+m$ & ENTV & $P V / T I V$ & 1 & $\mathrm{PI/HIV}$ & ديلن اطرافيان در حال مرى & \\
\hline & & & r/RTq & if & $m e / 1 \mu r$ & مرى خود & \\
\hline & & & F/quA & if & EQ/IT" & ديلن خود درحال مرى & \\
\hline & & & F/afY & if & $9+91$. & هرك اطرافيان & \\
\hline & & & g/Ifi & if & $1 \Delta / 21$ & ديلن اطرافيان در حال مرى & \\
\hline & & & & r. & MISTgl... & مركى خود & \\
\hline & & & & r. & $r . \circ A \% \ldots$ & ديدن خُود درحال مرى & \\
\hline & & & & r. & $|M \Delta \Delta| / \ldots$ & هرى اطرافيان & جمع \\
\hline & & & & r. & $199 \mathrm{~W} / \mathrm{0.}$ & ديلن اطرافيان در حال مرى & \\
\hline
\end{tabular}

几

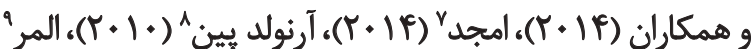

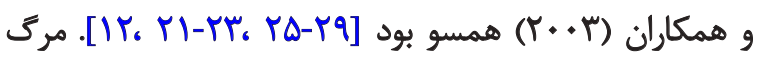

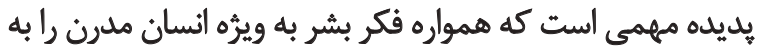

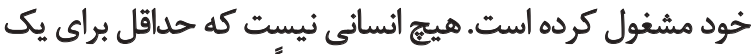

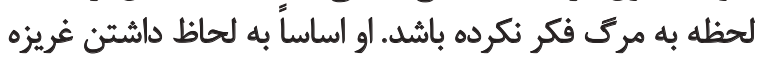

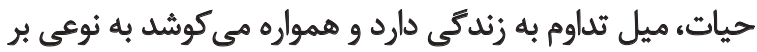

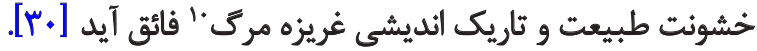

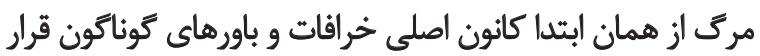

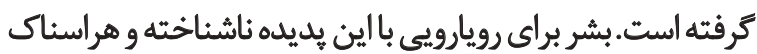
به مذهب و فلسفه يناه برده و با توسل به علم و هنر تحمل آن بآن

\section{Amjad}

8. Arnold Pyne

9. Elmer

10. Death instinct
سالمندان شهر سنندج بود.نتايج يُروهش حاضر نشان داد آموزش مؤلفههاى هوش معنوى باعث كاهش اضطراب مركى ساضئ سالمندان

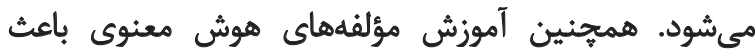

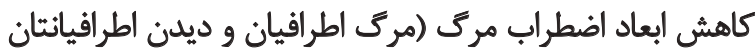

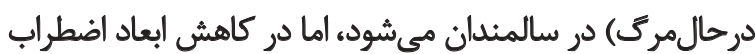

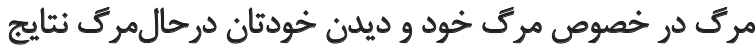
معنادارى مشاهده نشد.

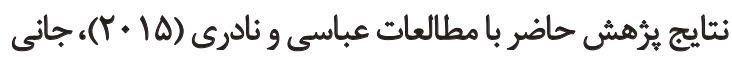

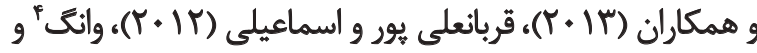

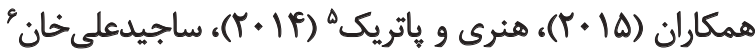


و خليفه و جانشين خدابودن در زمين، أكاه و تحت آموزش برد

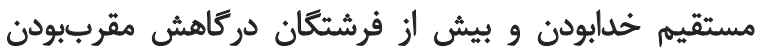

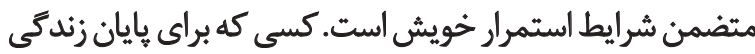

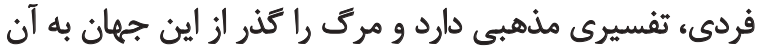

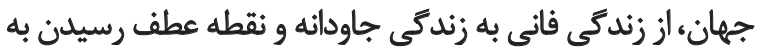

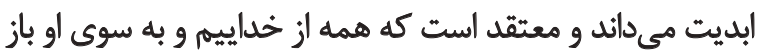

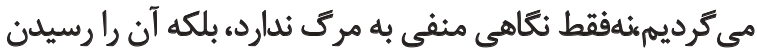
و وييوستن به خالق خود ثلقى مى كند [IIIT].

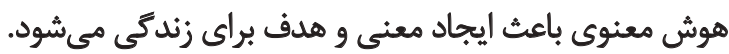

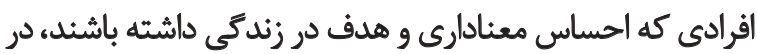

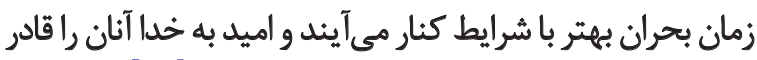

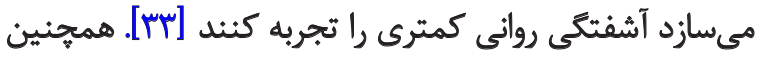

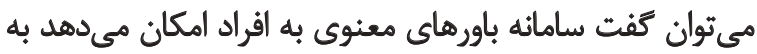

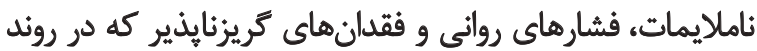

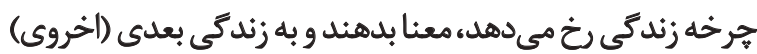

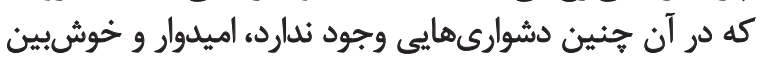

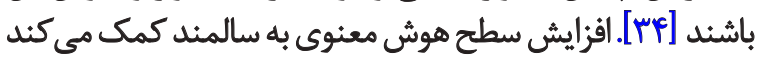

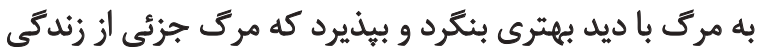

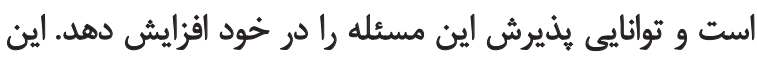

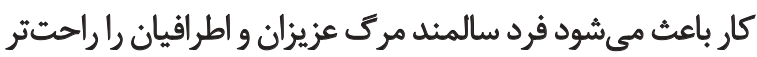

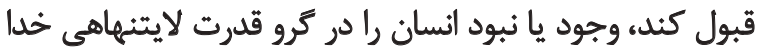

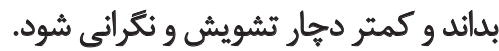

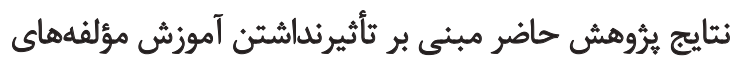

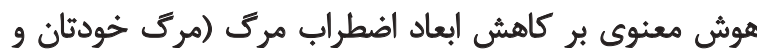

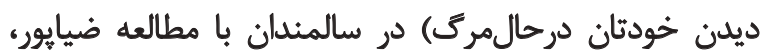

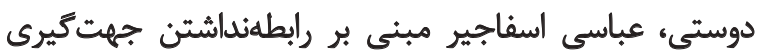

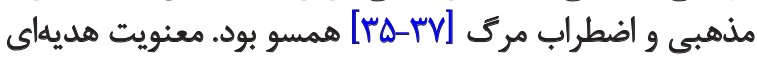

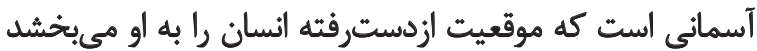

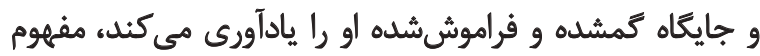

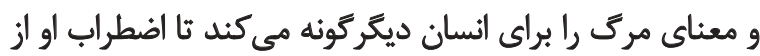

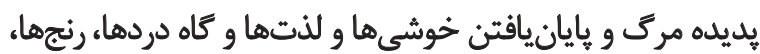

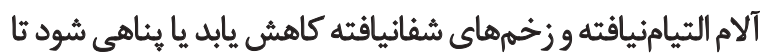

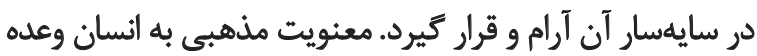

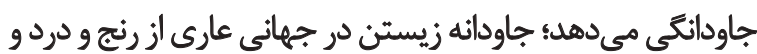

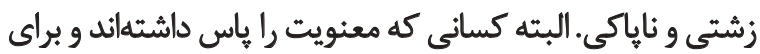

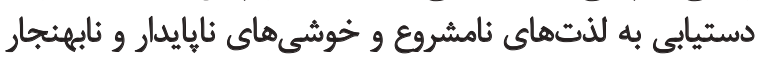

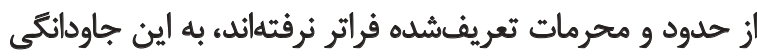

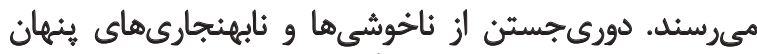

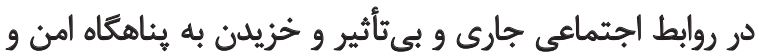

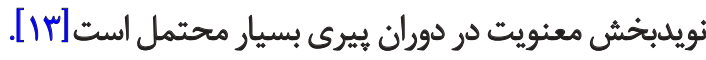

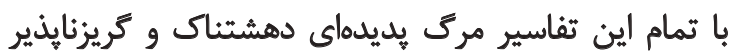

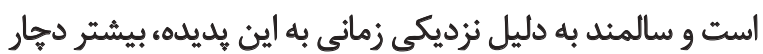

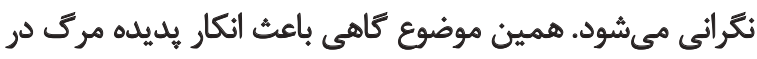

را آسانتر كرده است. معنويت ترس از مرك را راهش مئ ميدهد

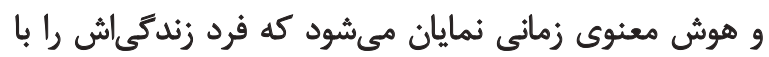

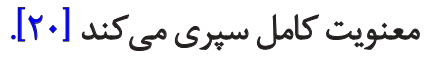
افرادى كه هوش معنوى بالاترى دارند، به سه دليل سلامت

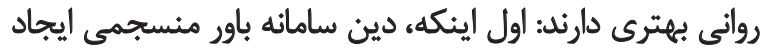

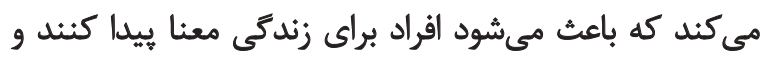

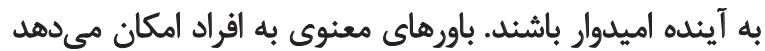

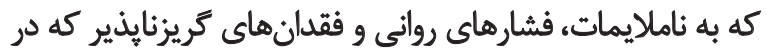

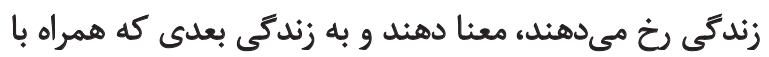

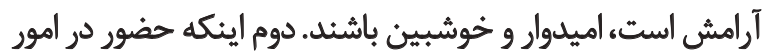

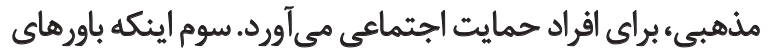

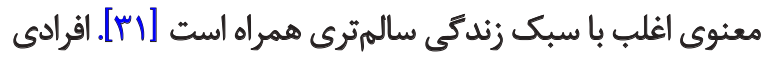

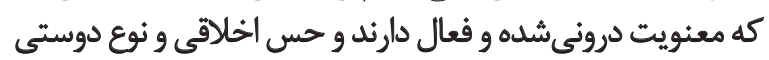

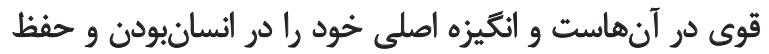

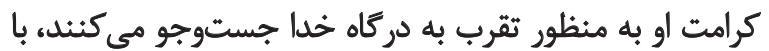

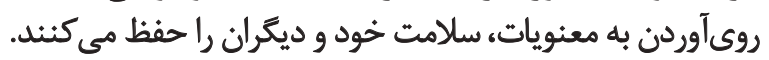

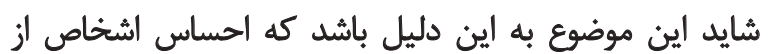

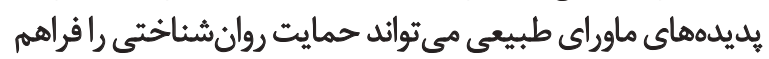

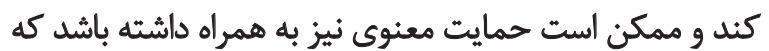

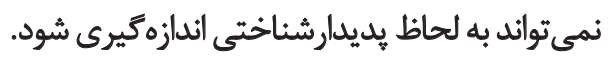

به دليل ماهيت متعالى تجربههاي معنوى، افراد با باورهاى

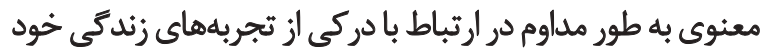

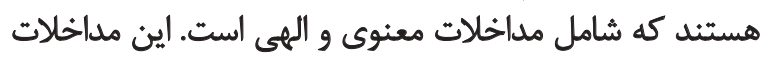

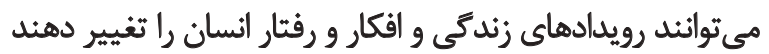

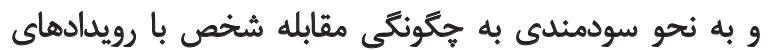

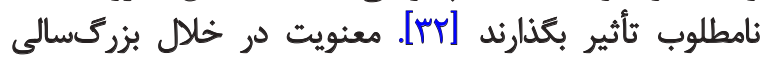

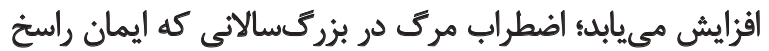

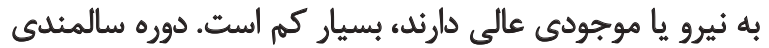

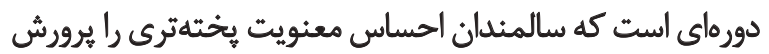

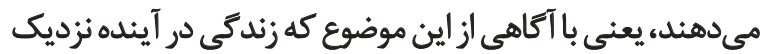

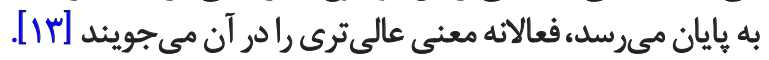

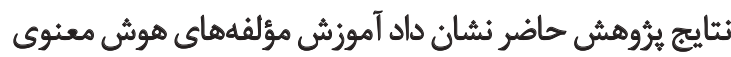

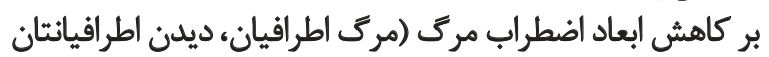

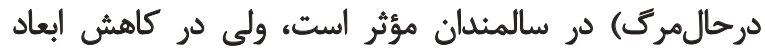

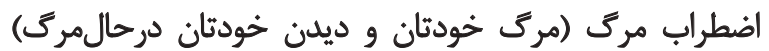

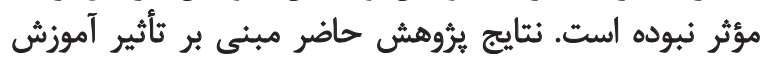

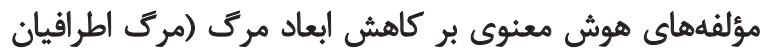

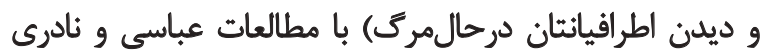

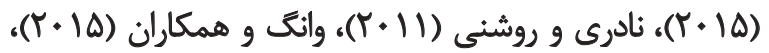

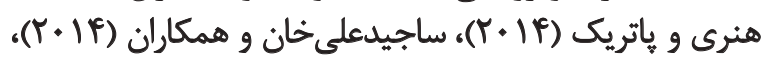

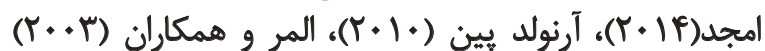

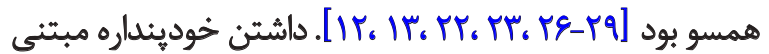

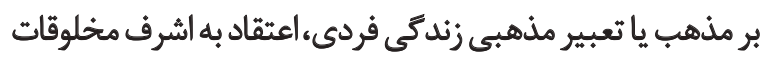


او مىشود و معنويت و آموزههاى دينى توان مقابله با اين تفكر

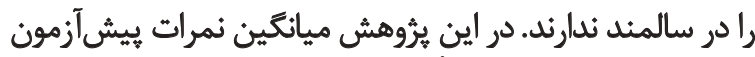

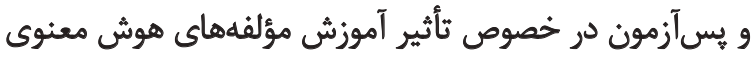

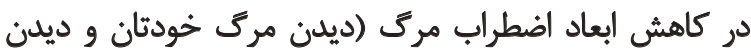

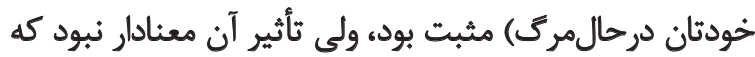

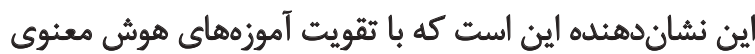

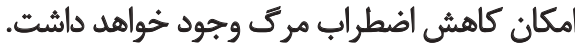

از جمله محدوديتهاى تحقيق حاضر اين بود كه به دليل

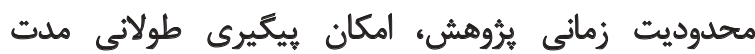

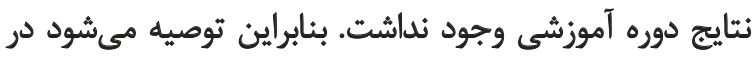

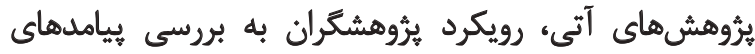

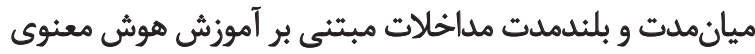

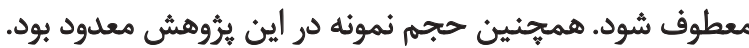

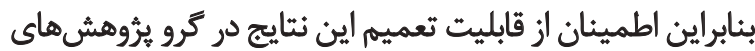

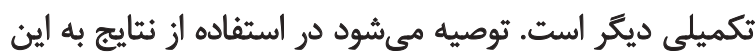
محلدوديت نيز توجه شئود. تتيجهئيرىنهايى

نتايج يروهش حاضر نشان داد آموزش مؤلفههاى هوش

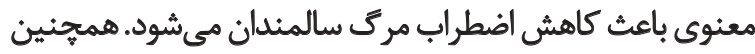

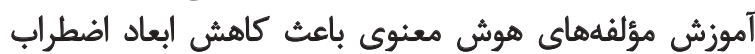

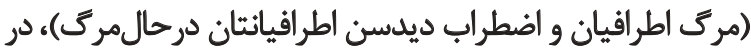

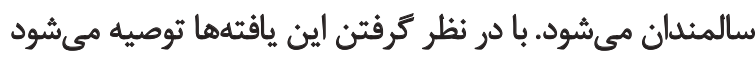

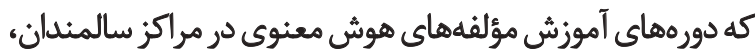

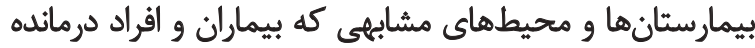

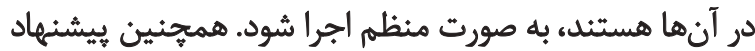

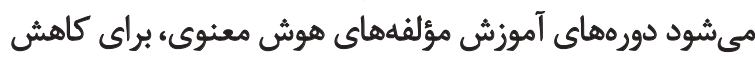

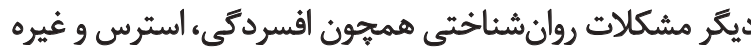

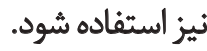

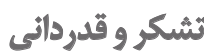

مؤلفان بر خود لازم مي دانند كه ضمن سياسكزارى از مديريت

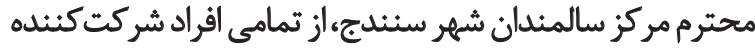

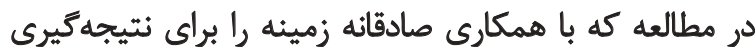
دقيقتر فراهم كردند، صميمانه تشكر و قدردانى كنيند.

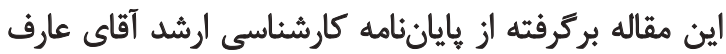

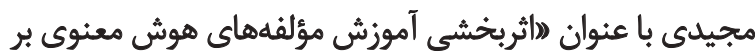

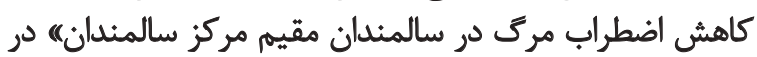

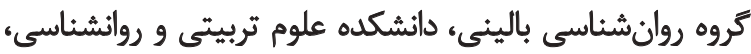
دانشكاه آزاد اسلامى واحد سنئدج است. 


\section{References}

[1] Hojati H, Sharifnia H, Hoseinalipour S, Akhundzade G, Asayesh $\mathrm{H}$. [The effect of reminiscence on the amount of group self-esteem and life satisfaction of the elderly (Persian)]. Journal of Nursing and Midwifery Urmia University of Medical Sciences. 2011; 9(5):350-6.

[2] Riedel Heller SG, Busse A, Angermeyer MC. The state of mental health in old age across the 'old'European Uniona systematic review. Acta Psychiatrica Scandinavica. 2006; 113(5):388-401. doi: 10.1111/j.1600-0447.2005.00632.x

[3] Parmar D, Williams G, Dkhimi F. Enrolment of older people in social health protection programs in West Africa e Does social exclusion play a part. Social Science \& Medicine. 2014; 119:36-44.

[4] Statistical Center of Iran. [General census of population and housing (Persian)]. Tehran: Statistical Center of Iran; 2016.

[5] Tsai FJ, Motamed S, Rougemont A. The protective effect of taking care of grandchildren on elders' mental health? Associations between changing patterns of intergenerational exchanges and the reduction of elders' loneliness and depression between 1993 and 2007 in Taiwan. BMC Public Health. 2013; 13(1). doi: 10.1186/1471-2458-13-567

[6] Bøen H, Dalgard OS, Johansen R, Nord E. A randomized controlled trial of a senior centre group programme for increasing social support and preventing depression in elderly people living at home in Norway. BMC Geriatrics. 2012; 12(1). doi: 10.1186/1471-2318-12-20

[7] Phabphal K, Geater A, Limapichat K, Sathirapanya P, Setthawatcharawanich S. Risk factors of recurrent seizure, co-morbidities, and mortality in new onset seizure in elderly. Seizure. 2013; 22(7):577-80. Doi: 10.1016/j.seizure.2013.04.009

[8] Bährer-Kohler S, Hemmeter U. Aspects of mental health care provision of the elderly in Switzerland. Geriatric Mental Health Care. 2013; 1(1):11-9. doi: 10.1016/j. gmhc.2012.11.002

[9] Berk, LE. Child development [Seyed Mohammadi Y, Persian trans]. Tehran: Arasbaran; 2008.

[10] Salehi F, Mohsenzade F, Arefi M. [Prevalence of death anxiety in patients with breast cancer in Kermanshah (Persian)]. Iranian Journal of Breast Disease. 2016; 8(4):34-41.

[11] Caplan H. Psychiatry [M. Sadeghi, M. Sadeghi, S. Mohsenifar, V. Shariat, A. Farhoodian, V. Sharifi, Persian Trans]. Tehran: Baraye Farda; 2002.

[12] Abbasi Z, Naderi F. [The relationship between spiritual intelligence, meaningful life and death anxiety and depression in cancer patients in Ahvaz (Persian)]. Paper presented at the $2^{\text {nd }}$ National Conference and the $1^{\text {st }}$ International Conference on New Research in the Humanities. 12 June 2015, Tehran, Iran

[13] Naderi F, Roshani Kh. [The relationship between spiritual intelligence, social intelligence and death anxiety in the elderly (Persian)]. Woman and Culture. 2011; 2(6):55-67.

[14] Gobarybonab B, Salimi M, Sliani L, noorimoghadam S. [Spiritual intelligence (Persian)]. Research Quarterly in Islamic Theology (Kalam) and Religious Studies. 2007; 3(10):125-47.
[15] Abedini Y, Brat Dastjerdi N. [The relation between religiosity and spiritual intelligence and their effect on students' academic achievement (Persian)]. New Educational Approaches. 2014; 9(1):37-52.

[16] King DB. Rethinking claims of spiritual intelligence: A definition, model, \& measure (MA thesis). Peterborough, Ontario: Trent university; 2008.

[17] Alavi S. [The relationship between spiritual intelligence and attitude to love and orientation to the relationship before marriage in Isfahan Industrial University (Persian)] [MA thesis]. Isfahan: Islamic Azad University; 2011.

[18] Abdollahzadeh H. [Construction and standardization of spiritual intelligence questionnaire (Persian)]. Tehran: Psychometric; 2009.

[19] Salajegheh S, Raghibi M. [The effect of combined therapy of spiritual-cognitive group therapy on death anxiety in patients with cancer (Persian)]. Journal of Shahid Sadoughi University of Medical Sciences. 2014; 22(2):1130-9.

[20] Bairami M, Mohammad Panah Ardakan A, Faroughi P, Ghanei M. [Prediction of death anxiety based on spiritual intelligence of students in Ardakan university and seminary (Persian)]. Culture in the Islamic University. 2015; 5(1):21-37.

[21] Jani S, Molaee M, Jangi S, Pooresmaili A. [Effectiveness of cognitive therapy based on religious believes on death anxiety, social adjustment and subjective well-being in the cancer patients (Persian)]. Journal of Medical Sciences. 2014; 22(5):94-103.

[22] Henrie J, Patrick JH. Religiousness, religious doubt, and death anxiety. The International Journal of Aging and Human Development. 2014; 78(3):203-27. doi: 10.2190/ ag.78.3.a

[23] Amjad A. Death anxiety as a function of age and religiosity. Journal of Applied Environmental and Biological Sciences. 2014; 4(9):333-41.

[24] Naderi F, Esmaeli E. [The association Between Death Anxiety, Suicide ideation and wellbeing in Ahvaz I.A.U Students (Persian)]. Journal of Social Psychology. 2009; 2(8):35-52.

[25] Naderi F, Esmaeli E. [The association between death anxiety, suicide ideation and wellbeing in Ahvaz I.A.U students (Persian)]. Journal of Social Psychology. 2009; 2(8):35-52.

[26] Qorbanalipoor M, Esmaeili A. [The effectiveness of the therapy on the anxiety of death in the elderly (Persian)]. Counseling Culture and Psychotherapy. 2012; 3(9):53-68.

[27] Wong LP, Fung HH, Jiang D. Associations between religiosity and death attitudes: Different between Christians and Buddhists. Psychology of Religion and Spirituality. 2015; 7(1):70-9. doi: 10.1037/a0037993

[28] Sajid Ali Khan K, Vijayshri R, Farooqi FS. A study of religiosity in relation to spirituality and anxiety. International Journal of Advancements in Research Technology. 2014; 3(4):269-85.

[29] Pyne DA. A model of religion and death. The Journal of Socio-Economics. 2010; 39(1):46-54. doi: 10.1016/j.socec.2009.08.003 
[30] Elmer LD, MacDonald DA, Friedman HL. Transpersonal psychology, physical health, and mental health: Theory, research, and practice. The Humanistic Psychologist. 2003; 31(2-3):159-81. doi: 10.1080/08873267.2003.9986929

[31] Azar M, Nohee S, Shafiee Kandjani AR. [Suicide (Persian)]. Tehran: Arjmand; 2006.

[32] Mabe PA, Josephson AM. Child and adolescent psychopathology: Spiritual and religious perspectives. Child and Adolescent Psychiatric Clinics of North America. 2004; 13(1):111-25. doi: 10.1016/s1056-4993(03)00091-9

[33] Carr A. Positive psychology: The science of happiness and human strengths. New York: Routledge; 2011.

[34] Mabe PA, Josephson AM. Child and adolescent psychopathology: spiritual and religious perspectives. Child and Adolescent Psychiatric Clinics of North America. 2004; 13(1):111-25. doi: 10.1016/s1056-4993(03)00091-9

[35] Sim TN, Loh BSM. Attachment to god: Measurement and dynamics. Journal of Social and Personal Relationships. 2003; 20(3):373-89. doi: 10.1177/0265407503020003006

[36] Smucker CJ. Nursing, healing and spirituality. Complementary Therapies in Nursing and Midwifery. 1998; 4(4):95-7. doi: 10.1016/s1353-6117(98)80040-1

[37] Ziapour SS, Dusti Y, AbbasiAsfajir A. Correlation between religious orientation and death anxiety. Journal of Psychology \& Behavioral Studies. 2014; 2(1):20-29. 
